\title{
A Numeric - Analytic Method for Fractional Order Nonlinear PDE's With Modified Riemann-Liouville Derivative by Means of Fractional Variational Iteration Method
}

\section{Mehmet Merdan*}

Gümüşhane University, Department of Mathematics Engineering, 29100-Gümüşhane, Turkey

\begin{abstract}
In this article, an approximate analytical solution of fractional order nonlinear PDE's with modified RiemannLiouville derivative was obtained with the help of fractional variational iteration method (FVIM). It is showed that the solutions obtained by the FVIM are reliable and effective method for strongly nonlinear partial equations with modified Riemann-Liouville derivative. The solutions of our model equation can also be obtained from the known forms of the series solutions.
\end{abstract}

Keywords: Fractional variational iteration method; Fractional KDV; $\mathrm{K}(2,2)$ and $\mathrm{mKDV}$ equation; Riemann-Liouville derivative, Burgers equation, Klein-Gordon equation

\section{Introduction}

It is known that various problems in electrical networks, control theory of dynamical systems, probability and statistics, electrochemistry of corrosion, chemical physics, optics, engineering, acoustics, material science and signal processing can be successfully modeled by linear or nonlinear fractional order differential equations. Methods of solutions of problems for fractional differential equations have been studied extensively by many researchers [1-8]

The variational iteration method (VIM), which proposed by JiHuan He [9], was successfully applied to autonomous ordinary and partial differential equations and other fields. Ji-Huan He [10] was the first to apply the variational iteration method to fractional differential equations.

Fractional convection-diffusion equation with nonlinear source term solved by Momani and Yildirim [11], space-time fractional advection-dispersion equation by Yildirim and Kocak [12], fractional Zakharov-Kuznetsov equations by Yildirim and Gulkanat [13], integro-differential equation by El-Shahed [14], non-Newtonian flow by Siddiqui et al. [15], fractional PDEs in fluid mechanics by Yildirim [16], fractional Schrödinger equation [17,18]. Different fractional partial differential equations have been studied and solved including the space-time fractional diffusion-wave equation [19-21], the fractional advection-dispersion equation [22,23], the fractional telegraph equation [24], the fractional $\mathrm{KdV}$ equation [25] and the linear inhomogeneous fractional partial differential equations [26]. Recently, a new modified Riemann-Liouville left derivative is suggested by G. Jumarie [27-31]. The most recently, a new application of Fractional Variational Iteration Method (FVIM) for solving non-linear fractional coupled- KDV equations with modified Riemann-Liouville derivative performed by Merdan et al. [32]. Roul [33] applied the fractional VIM for obtaining the exact and approximate analytical solutions of time fractional biological population models.

The aim of this paper is to extend the application of the variational iteration method method to solve fractional KDV, $\mathrm{K}(2,2)$, mKDV equation and some fractional partial equations in fluid mechanics with modified Riemann-Liouville derivative.

This paper is organized as follows:

In Basic definitions section, we gave definitions related to the fractional calculus theory briefly. In Fractional variational iteration method section, we define the solution procedure of the fractional variational iteration method. To show in efficiency of this method, we give the implementation of the FVIM for the fractional KDV, K $(2,2), \mathrm{mKDV}$ equation and some fractional partial equations in fluid mechanics with modified Riemann-Liouville derivative and numerical results in Applications section. The conclusions are then given at the last.

\section{Basic Definitions}

Here, we give some basic definitions and properties of fractional calculus theory which will be used in this paper [1,2,27-37].

\section{Definition 1}

Fractional derivative is defined as the following limit form [27-29]

$$
f^{(\alpha)}=\lim _{h \rightarrow 0} \frac{\Delta^{\alpha}[f(x)-f(0)]}{h^{\alpha}}
$$

This definition is close to the standard definition of derivatives (calculus for beginners), and as a direct result, the $\alpha$ th derivative of a constant, $0<\alpha<1$, is zero.

\section{Definition 2}

The left-sided Riemann-Liouville fractional integral operator of order $\alpha \geq 0$, of a function $f \in C_{\mu}, \mu \geq-1$ is defined as

$$
\begin{aligned}
& J_{a}^{\alpha} f(x)=\frac{1}{\Gamma(\alpha)} \int_{a}^{x}(x-\tau)^{\alpha-1} f(\tau) d \tau, \\
& \text { for } \alpha>0, x>0 \text { and } J_{a}^{0} f(x)=f(x) .
\end{aligned}
$$

The properties of the operator $J^{\alpha}$ can be found in $[1,2,34]$.

*Corresponding author: Mehmet Merdan, Gümüşhane University, Department of Mathematics Engineering, 29100-Gümüşhane, Turkey, E-mail: mmerdan@gumushane.edu.tr

Received May 16, 2012; Accepted June 11, 2012; Published June 15, 2012

Citation: Merdan M (2012) A Numeric-Analytic Method for Fractional Order Nonlinear PDE's With Modified Riemann-Liouville Derivative by Means of Fractional Variational Iteration Method. J Appl Computat Math 1:113. doi:10.4172/ 2168-9679.1000113

Copyright: (c) 2012 Merdan M. This is an open-access article distributed unde the terms of the Creative Commons Attribution License, which permits unrestricted use, distribution, and reproduction in any medium, provided the original author and source are credited. 


\section{Definition 3}

The modified Riemann-Liouville derivative [27-29] is defined as

$$
{ }_{0} D_{\alpha}^{x} f(x)=\frac{1}{\Gamma(m-\alpha)} \frac{d^{m}}{d x^{m}} \int_{x}^{0}(x-\tau)^{m-\alpha}(f(\tau)-f(0)) d \tau,
$$

where $x \in[0,1], m-1 \leq \alpha<m$ and $m \geq 1$.

\section{Definition 4}

Fractional derivative of compounded functions [27-29] is defined as

$$
d^{\alpha} f \cong \Gamma(1+\alpha) d f, 0<\alpha<1
$$

\section{Definition 5}

The integral with respect to $(d x)^{\alpha}$ [27-29] is defined as the solution of the fractional differential equation

$$
d y \cong f(x)(d x)^{\alpha}, \quad x \geq 0, \quad y(0)=0, \quad 0<\alpha<1
$$

\section{Lemma 1}

Let $f(x)$ denote a continuous function [27-29] then the solution of the Eq. (5) is defined as

$$
y=\int_{0}^{x} f(\tau)(d \tau)^{\alpha}=\alpha \int_{0}^{x}(x-\tau)^{\alpha} f(\tau) d \tau, 0<\alpha<1
$$

For example $f(x)=x^{\beta}$ in Eq. (6) one obtains

$$
\int_{0}^{x} \tau^{\beta}(d \tau)^{\alpha}=\frac{\Gamma(\beta+1) \Gamma(\alpha+1)}{\Gamma(\alpha+\beta+1)} x^{\beta+\alpha}, 0<\alpha<1
$$

\section{Definition 6}

Assume that the continuous function $f: R \rightarrow R, x \rightarrow f(x)$ has a fractional derivative of order $k \alpha$, for any positive integer k and any $\alpha$ , $0<\alpha \leq 1$; then the following

equality holds, which is

$$
f(x+h)=\sum_{k=0}^{\infty} \frac{h^{\alpha k}}{\alpha k !} f^{\alpha k}(x), \quad 0<\alpha \leq 1 .
$$

On making the substitution $h \rightarrow x$ and $x \rightarrow 0$ we obtain the fractional Mc-Laurin series

$$
f(x)=\sum_{k=0}^{\infty} \frac{x^{\alpha k}}{\alpha k !} f^{\alpha k}(0), \quad 0<\alpha \leq 1 .
$$

\section{Fractional Variational Iteration Method}

To describe the solution procedure of the fractional variational iteration method, we consider the following fractional differential equation [34]:

$$
\begin{aligned}
& D_{t}^{\alpha} u(x, t)=G\left(u(x, t), \frac{\partial u(x, t)}{\partial x}, \frac{\partial^{2} u(x, t)}{\partial x^{2}}, \frac{\partial^{3} u(x, t)}{\partial x^{3}}\right), \quad t>0, \quad x \in R, \\
& u(x, 0)=f(x), \quad \alpha>0
\end{aligned}
$$

According to the VIM, we can build a correct functional for Eq. (4) as follows

$$
\begin{aligned}
& u_{n+1}(x, t)=u_{n}(x, t)+I^{\alpha}\left[\lambda(x, t)\left(\frac{\partial^{\alpha} u_{n}(x, t)}{\partial t^{\alpha}}-G\left(u(x, t), \frac{\partial u(x, t)}{\partial x}, \frac{\partial^{2} u(x, t)}{\partial x^{2}}, \frac{\partial^{3} u(x, t)}{\partial x^{3}}\right)\right)\right] \\
& u_{n+1}(x, t)=u_{n}(x, t)+ \\
& \frac{1}{\Gamma(\alpha)} \int_{0}^{\int}(t-\tau)^{\alpha-1} \lambda(x, \tau)\left(\frac{\partial^{\alpha} u_{n}(x, \tau)}{\partial \tau^{\alpha}}-G\left(u(x, \tau), \frac{\partial u(x, \tau)}{\partial x}, \frac{\partial^{2} u(x, \tau)}{\partial x^{2}}, \frac{\partial^{3} u(x, \tau)}{\partial x^{3}}\right)\right) d \tau
\end{aligned}
$$

Using Eq. (5), we obtain a new correction functional

$$
\begin{aligned}
u_{n+1}(x, t)= & u_{n}(x, t)+ \\
& \frac{1}{\Gamma(\alpha+1)} \int_{0}^{1} \lambda(x, \tau)\left(\frac{\partial^{\alpha} u_{n}(x, \tau)}{\partial \tau^{\alpha}}-G\left(u(x, \tau), \frac{\partial u(x, \tau)}{\partial x}, \frac{\partial^{2} u(x, \tau)}{\partial x^{2}}, \frac{\partial^{3} u(x, \tau)}{\partial x^{3}}\right)\right)(d \tau)^{\alpha}
\end{aligned}
$$

It is obvious that the sequential approximations $u_{k}, k \geq 0$ can be established by determining $\lambda$, a general Lagrange's multiplier, which can be identified optimally with the variational theory. The function $\tilde{u}_{n}$ is a restricted variation which means $\delta \tilde{u}_{n}=0$. Therefore, we first designate the Lagrange multiplier $\lambda$ that will be identified optimally via integration by parts. The successive approximations $u_{n+1}(x, t), n \geq 0$ of the solution $u(x, t)$ will be readily obtained upon using the obtained Lagrange multiplier and by using any selective function $u_{0}$. The initial values are usually used for choosing the zeroth approximation $u_{0}$ . With $\lambda$ determined, then several approximations $u_{k}, k \geq 0$ follows immediately [35]. Consequently, the exact solution may be procured by using

$$
u(x, t)=\lim _{n \rightarrow \infty} u_{n}(x, t)
$$

\section{Applications}

In this section, we present the solution of nonlinear fractional partial differential equations as the applicability of FVIM.

\section{Example 1}

Consider the fractional KDV equation where $0<\alpha \leq 1$

$$
D_{t}^{\alpha} u-3\left(u^{2}\right)_{x}+u_{x x x}=0
$$

With initial conditions

$$
u(x, 0)=6 x,[36] \text {. }
$$

Construction the following functional:

$$
\begin{aligned}
& u_{n+1}(x, t)=u_{n}(x, t)+\frac{1}{\Gamma(\alpha+1)} \int_{0}^{t} \lambda(x, \tau) \\
& \left\{\frac{\partial^{\alpha} u_{n}(x, \tau)}{\partial \tau^{\alpha}}-6 u_{n}(x, \tau) \frac{\partial u_{n}(x, \tau)}{\partial x}+\frac{\partial^{3} u_{n}(x, \tau)}{\partial x^{3}}\right\}(d \tau)^{\alpha}
\end{aligned}
$$

We have

$$
\begin{aligned}
& \delta u_{n+1}(x, t)=\delta u_{n}(x, t)+\frac{1}{\Gamma(\alpha+1)} \delta \int_{0}^{t} \lambda(x, \tau) \\
& \left\{\frac{\partial^{\alpha} u_{n}(x, \tau)}{\partial \tau^{\alpha}}-6 u_{n}(x, \tau) \frac{\partial u_{n}(x, \tau)}{\partial x}+\frac{\partial^{3} u_{n}(x, \tau)}{\partial x^{3}}\right\}(d \tau)^{\alpha} \\
& =\delta u_{n}+\left.\lambda \delta u_{n}\right|_{\tau=t}-\frac{1}{\Gamma(\alpha+1)} \int_{0}^{t} \frac{\partial^{\alpha} \lambda(x, \tau)}{\partial \tau^{\alpha}} \delta u_{n}(x, \tau)(d \tau)^{\alpha}
\end{aligned}
$$

Similarly, we can get the coefficients of $\delta u_{n}$ to zero:

$$
1+\left.\lambda(x, \tau)\right|_{\tau=t}=0, \quad \frac{\partial^{\alpha} \lambda(x, \tau)}{\partial \tau^{\alpha}}=0
$$


The generalized Lagrange multiplier can be identified by the above equations,

$$
\lambda(x, t)=-1 \text {. }
$$

Substituting Eq. (19) into Eq. (16) produces the iteration formulation as follows

$$
\begin{aligned}
& u_{n+1}(x, t)=u_{n}(x, t)-\frac{1}{\Gamma(\alpha+1)} \\
& \int_{0}^{t}\left\{\frac{\partial^{\alpha} u_{n}(x, \tau)}{\partial \tau^{\beta}}-6 u_{n}(x, \tau) \frac{\partial u_{n}(x, \tau)}{\partial x}+\frac{\partial^{3} u_{n}(x, \tau)}{\partial x^{3}}\right\}(d \tau)^{\alpha}
\end{aligned}
$$

Taking the initial value $u_{0}(x, t)=u_{0}(x, 0)=6 x$, we can derive

$$
\begin{aligned}
& u_{1}(x, t)=u_{0}(x, t)-\frac{1}{\Gamma(\alpha+1)} \\
& \int_{0}^{t}\left\{\frac{\partial^{\alpha} u_{0}(x, \tau)}{\partial \tau^{\beta}}-6 u_{0}(x, \tau) \frac{\partial u_{0}(x, \tau)}{\partial x}+\frac{\partial^{3} u_{0}(x, \tau)}{\partial x^{3}}\right\}(d \tau)^{\alpha} \\
& u_{1}(x, t)=6 x+\frac{6^{3} x t^{\alpha}}{\Gamma(\alpha+1)}, \\
& u_{2}(x, t)=6 x+\frac{6^{3} x t^{\alpha}}{\Gamma(\alpha+1)}+\frac{2^{*} 6^{5} x t^{2 \alpha}}{\Gamma(2 \alpha+1)}+\frac{6^{7} \Gamma(2 \alpha+1) x t^{3 \alpha}}{\Gamma^{2}(\alpha+1) \Gamma(3 \alpha+1)} .
\end{aligned}
$$

For the special case $\alpha=1$ is [36]

$$
\begin{aligned}
u(x, t)=\operatorname{Lim}_{n \rightarrow \infty} u_{n}(x, t) & =6 x\left(1+36 t+36^{2} t^{2}+36^{3} t^{3}+\cdots\right) \\
& =\frac{6 x}{1-36 t}
\end{aligned}
$$

\section{Example 2}

Consider the fractional $\mathrm{K}(2,2)$ equation where $0<\alpha \leq 1$

$D_{t}^{\alpha} u+\left(u^{2}\right)_{x}+\left(u^{2}\right)_{x x x}=0$

With initial conditions

$u(x, 0)=x,[36]$.

Construction the following functional:

$$
\begin{aligned}
& u_{n+1}(x, t)=u_{n}(x, t)+\frac{1}{\Gamma(\alpha+1)} \int_{0}^{t} \lambda(x, \tau) \\
& \left\{\begin{array}{l}
\frac{\partial^{\alpha} u_{n}(x, \tau)}{\partial \tau^{\alpha}}+2 u_{n}(x, \tau) \frac{\partial u_{n}(x, \tau)}{\partial x} \\
4 \frac{\partial u_{n}(x, \tau)}{\partial x} \frac{\partial^{2} u_{n}(x, \tau)}{\partial x^{2}}+2 u_{n}(x, \tau) \frac{\partial^{3} u_{n}(x, \tau)}{\partial x^{3}}
\end{array}\right\}(d \tau)^{\alpha}
\end{aligned}
$$

We have

$$
\begin{aligned}
& \delta u_{n+1}(x, t)=\delta u_{n}(x, t)+\frac{1}{\Gamma(\alpha+1)} \delta \int_{0}^{t} \lambda(x, \tau) \\
& \left\{\begin{array}{c}
\frac{\partial^{\alpha} u_{n}(x, \tau)}{\partial \tau^{\alpha}}+2 u_{n}(x, \tau) \frac{\partial u_{n}(x, \tau)}{\partial x} \\
4 \frac{\partial u_{n}(x, \tau)}{\partial x} \frac{\partial^{2} u_{n}(x, \tau)}{\partial x^{2}}+2 u_{n}(x, \tau) \frac{\partial^{3} u_{n}(x, \tau)}{\partial x^{3}}
\end{array}\right\}(d \tau)^{\alpha} \\
& =\delta u_{n}+\left.\lambda \delta u_{n}\right|_{\tau=t}-\frac{1}{\Gamma(\alpha+1)} \int_{0}^{t} \frac{\partial^{\alpha} \lambda(x, \tau)}{\partial \tau^{\beta}} \delta u_{n}(x, \tau)(d \tau)^{\alpha}
\end{aligned}
$$

Similarly, we can get the coefficients of $\delta u_{n}$ to zero:

$$
1+\left.\lambda(x, \tau)\right|_{\tau=t}=0, \quad \frac{\partial^{\alpha} \lambda(x, \tau)}{\partial \tau^{\alpha}}=0
$$

The generalized Lagrange multiplier can be identified by the above equations,

$$
\lambda(x, t)=-1
$$

Substituting Eq. (28) into Eq. (25) produces the iteration formulation as follows

$$
\begin{aligned}
& u_{n+1}(x, t)=u_{n}(x, t)-\frac{1}{\Gamma(\alpha+1)} \\
& \left\{\begin{array}{l}
\frac{\partial^{\alpha} u_{n}(x, \tau)}{\partial \tau^{\alpha}}+2 u_{n}(x, \tau) \frac{\partial u_{n}(x, \tau)}{\partial x} \\
4 \frac{\partial u_{n}(x, \tau)}{\partial x} \frac{\partial^{2} u_{n}(x, \tau)}{\partial x^{2}}+2 u_{n}(x, \tau) \frac{\partial^{3} u_{n}(x, \tau)}{\partial x^{3}}
\end{array}\right\}(d \tau)^{\alpha}
\end{aligned}
$$

Taking the initial value $u_{0}(x, t)=u_{0}(x, 0)=x$, we can derive

$$
u_{1}(x, t)=u_{0}(x, t)-\frac{1}{\Gamma(\alpha+1)}
$$$$
\left\{\begin{array}{l}
\frac{\partial^{\alpha} u_{0}(x, \tau)}{\partial \tau^{\alpha}}+2 u_{0}(x, \tau) \frac{\partial u_{0}(x, \tau)}{\partial x} \\
4 \frac{\partial u_{0}(x, \tau)}{\partial x} \frac{\partial^{2} u_{0}(x, \tau)}{\partial x^{2}}+2 u_{0}(x, \tau) \frac{\partial^{3} u_{0}(x, \tau)}{\partial x^{3}}
\end{array}\right\}(d \tau)^{\alpha}
$$

$u_{1}(x, t)=x-\frac{2 x t^{\alpha}}{\Gamma(\alpha+1)}$,

$u_{2}(x, t)=x-\frac{2 x t^{\alpha}}{\Gamma(\alpha+1)}+\frac{8 x t^{2 \alpha}}{\Gamma(2 \alpha+1)}+\frac{\Gamma(2 \alpha+1) 8 x t^{3 \alpha}}{\Gamma^{2}(\alpha+1) \Gamma(3 \alpha+1)}$.

For the special case $\alpha=1$ is [36]

$$
\begin{aligned}
u(x, t)=\operatorname{Lim}_{n \rightarrow \infty} u_{n}(x, t) & =6 x\left(1-2 t+4 t^{2}-8 t^{3}+\cdots\right) \\
& =\frac{6 x}{1+2 t} .
\end{aligned}
$$

\section{Example 3}

Consider the modified fractional KDV (mKDV) equation where $0<\alpha \leq 1$

$$
D_{t}^{\alpha} u+\frac{1}{2}\left(u^{2}\right)_{x}-u_{x x}=0,
$$

With initial conditions

$$
u(x, 0)=x,[36] .
$$

Construction the following functional:

$$
\begin{aligned}
& u_{n+1}(x, t)=u_{n}(x, t)+\frac{1}{\Gamma(\alpha+1)} \\
& \int_{0}^{t} \lambda(x, \tau)\left\{\begin{array}{l}
\frac{\partial^{\alpha} u_{n}(x, \tau)}{\partial \tau^{\alpha}} \\
+u_{n}(x, \tau) \frac{\partial u_{n}(x, \tau)}{\partial x}-\frac{\partial^{2} u_{n}(x, \tau)}{\partial x^{2}}
\end{array}\right\}(d \tau)^{\alpha}
\end{aligned}
$$


We have

$$
\begin{aligned}
& \delta u_{n+1}(x, t)=\delta u_{n}(x, t)+\frac{1}{\Gamma(\alpha+1)} \\
& \delta \int_{0}^{t} \lambda(x, \tau)\left\{\begin{array}{l}
\frac{\partial^{\alpha} u_{n}(x, \tau)}{\partial \tau^{\alpha}} \\
+u_{n}(x, \tau) \frac{\partial u_{n}(x, \tau)}{\partial x}-\frac{\partial^{2} u_{n}(x, \tau)}{\partial x^{2}}
\end{array}\right\}(d \tau)^{\alpha} \\
& =\delta u_{n}+\left.\lambda \delta u_{n}\right|_{\tau=t}-\frac{1}{\Gamma(\alpha+1)} \int_{0}^{t} \frac{\partial^{\alpha} \lambda(x, \tau)}{\partial \tau^{\beta}} \delta u_{n}(x, \tau)(d \tau)^{\alpha}
\end{aligned}
$$

Similarly, we can get the coefficients of $\delta u_{n}$ to zero:

$$
1+\left.\lambda(x, \tau)\right|_{\tau=t}=0, \quad \frac{\partial^{\alpha} \lambda(x, \tau)}{\partial \tau^{\alpha}}=0
$$

The generalized Lagrange multiplier can be identified by the above equations,

$$
\lambda(x, t)=-1
$$

Substituting Eq. (37) into Eq. (34) produces the iteration formulation as follows

$$
\begin{aligned}
& u_{n+1}(x, t)=u_{n}(x, t)-\frac{1}{\Gamma(\alpha+1)} \\
& \int_{0}^{t}\left\{\frac{\partial^{\alpha} u_{n}(x, \tau)}{\partial \tau^{\alpha}}+u_{n}(x, \tau) \frac{\partial u_{n}(x, \tau)}{\partial x}-\frac{\partial^{2} u_{n}(x, \tau)}{\partial x^{2}}\right\}(d \tau)^{\alpha}
\end{aligned}
$$

Taking the initial value $u_{0}(x, t)=u_{0}(x, 0)=x$, we can derive

$$
\begin{aligned}
& u_{1}(x, t)=u_{0}(x, t)-\frac{1}{\Gamma(\alpha+1)} \\
& \int_{0}^{t}\left\{\frac{\partial^{\alpha} u_{0}(x, \tau)}{\partial \tau^{\alpha}}+u_{0}(x, \tau) \frac{\partial u_{0}(x, \tau)}{\partial x}-\frac{\partial^{2} u_{0}(x, \tau)}{\partial x^{2}}\right\}(d \tau)^{\alpha} \\
& u_{1}(x, t)=x-\frac{x t^{\alpha}}{\Gamma(\alpha+1)}, \\
& u_{2}(x, t)=x-\frac{x t^{\alpha}}{\Gamma(\alpha+1)}+\frac{2 x t^{2 \alpha}}{\Gamma(2 \alpha+1)}-\frac{\Gamma(2 \alpha+1) x t^{3 \alpha}}{\Gamma^{2}(\alpha+1) \Gamma(3 \alpha+1)} .
\end{aligned}
$$

For the special case $\alpha=1$ is

$$
\begin{aligned}
u(x, t)=\operatorname{Lim}_{n \rightarrow \infty} u_{n}(x, t) & =x\left(1-2 t+4 t^{2}-8 t^{3}+\cdots\right) \\
& =\frac{x}{1+2 t} .
\end{aligned}
$$

\section{Example 4}

In this example we consider one-dimensional linear inhomogeneous fractional Burgers equation [37] where $0<\alpha \leq 1, t>0, x \in R$

$$
D_{t}^{\alpha} u+u_{x}-u_{x x}=\frac{2 t^{2-\alpha}}{\Gamma(3-\alpha)}+2 x-2,
$$

With initial conditions

$$
u(x, 0)=x^{2} .
$$

Construction the following functional:

$$
\begin{aligned}
& u_{n+1}(x, t)=u_{n}(x, t)+\frac{1}{\Gamma(\alpha+1)} \\
& \int_{0}^{t} \lambda(x, \tau)\left\{\begin{array}{l}
\frac{\partial^{\alpha} u_{n}(x, \tau)}{\partial \tau^{\alpha}}+\frac{\partial u_{n}(x, \tau)}{\partial x}-\frac{\partial^{2} u_{n}(x, \tau)}{\partial x^{2}} \\
-\frac{2 \tau^{2-\alpha}}{\Gamma(3-\alpha)}+2-2 x
\end{array}\right\}(d \tau)^{\alpha}
\end{aligned}
$$

We have

$$
\begin{aligned}
& \delta u_{n+1}(x, t)=\delta u_{n}(x, t)+\frac{1}{\Gamma(\alpha+1)} \\
& \delta \int_{0}^{t} \lambda(x, \tau)\left\{\begin{array}{l}
\frac{\partial^{\alpha} u_{n}(x, \tau)}{\partial \tau^{\alpha}}+\frac{\partial u_{n}(x, \tau)}{\partial x}-\frac{\partial^{2} u_{n}(x, \tau)}{\partial x^{2}} \\
-\frac{2 \tau^{2-\alpha}}{\Gamma(3-\alpha)}+2-2 x
\end{array}\right\}(d \tau)^{\alpha} \\
& =\delta u_{n}+\left.\lambda \delta u_{n}\right|_{\tau=t}-\frac{1}{\Gamma(\alpha+1)} \int_{0}^{t} \frac{\partial^{\alpha} \lambda(x, \tau)}{\partial \tau^{\beta}} \delta u_{n}(x, \tau)(d \tau)^{\alpha}
\end{aligned}
$$

Similarly, we can get the coefficients of $\delta u_{n}$ to zero:

$$
1+\left.\lambda(x, \tau)\right|_{\tau=t}=0, \quad \frac{\partial^{\alpha} \lambda(x, \tau)}{\partial \tau^{\alpha}}=0
$$

The generalized Lagrange multiplier can be identified by the above equations,

$$
\lambda(x, t)=-1
$$

substituting Eq. (46) into Eq. (43) produces the iteration formulation as follows

$$
\begin{aligned}
& u_{n+1}(x, t)=u_{n}(x, t)-\frac{1}{\Gamma(\alpha+1)} \\
& \int_{0}^{t}\left\{\begin{array}{l}
\frac{\partial^{\alpha} u_{n}(x, \tau)}{\partial \tau^{\alpha}}+\frac{\partial u_{n}(x, \tau)}{\partial x}-\frac{\partial^{2} u_{n}(x, \tau)}{\partial x^{2}} \\
-\frac{2 \tau^{2-\alpha}}{\Gamma(3-\alpha)}+2-2 x
\end{array}\right\}(d \tau)^{\alpha}
\end{aligned}
$$

Taking the initial value $u_{0}(x, t)=u_{0}(x, 0)=x^{2}$, we can derive

$$
\begin{aligned}
& u_{1}(x, t)=u_{0}(x, t)-\frac{1}{\Gamma(\alpha+1)} \\
& \int_{0}^{t}\left\{\begin{array}{l}
\frac{\partial^{\alpha} u_{0}(x, \tau)}{\partial \tau^{\alpha}}+\frac{\partial u_{0}(x, \tau)}{\partial x}-\frac{\partial^{2} u_{0}(x, \tau)}{\partial x^{2}} \\
-\frac{2 \tau^{2-\alpha}}{\Gamma(3-\alpha)}+2-2 x \\
=x^{2}+t^{2},
\end{array}\right.
\end{aligned}
$$

By the similar operations, we have

$$
\begin{aligned}
& u_{2}(x, t)=x^{2}+t^{2}, \\
& \vdots \\
& u_{n}(x, t)=x^{2}+t^{2} .
\end{aligned}
$$


Thus, the solution can be given a compact form:

$$
u(x, t)=\operatorname{Lim}_{n \rightarrow \infty} u_{n}(x, t)=x^{2}+t^{2}
$$

Which is the exact solution of Eq.(41) and in concordance with the conditions in Eq.(42).

\section{Example 5}

Consider the following one -dimensional linear inhomogeneous fractional wave equation [37] where $0<\alpha \leq 1, t>0, x \in R$

$$
D_{t}^{\alpha} u+u_{x}=\frac{t^{1-\alpha}}{\Gamma(2-\alpha)} \sin x+t \cos x,
$$

With initial conditions

$$
u(x, 0)=0 \text {. }
$$

Construction the following functional:

$$
\begin{aligned}
& u_{n+1}(x, t)=u_{n}(x, t)+\frac{1}{\Gamma(\alpha+1)} \\
& \int_{0}^{t} \lambda(x, \tau)\left\{\begin{array}{l}
\frac{\partial^{\alpha} u_{n}(x, \tau)}{\partial \tau^{\alpha}}+\frac{\partial u_{n}(x, \tau)}{\partial x} \\
-\frac{\tau^{1-\alpha}}{\Gamma(2-\alpha)} \sin x-\tau \cos x
\end{array}\right\}(d \tau)^{\alpha}
\end{aligned}
$$

We have

$$
\begin{aligned}
& \delta u_{n+1}(x, t)=\delta u_{n}(x, t)+\frac{1}{\Gamma(\alpha+1)} \\
& \delta \int_{0}^{t} \lambda(x, \tau)\left\{\begin{array}{l}
\frac{\partial^{\alpha} u_{n}(x, \tau)}{\partial \tau^{\alpha}}+\frac{\partial u_{n}(x, \tau)}{\partial x} \\
-\frac{\tau^{1-\alpha}}{\Gamma(2-\alpha)} \sin x-\tau \cos x
\end{array}\right\}(d \tau)^{\alpha} \\
& =\delta u_{n}+\left.\lambda \delta u_{n}\right|_{\tau=t}-\frac{1}{\Gamma(\alpha+1)} \int_{0}^{t} \frac{\partial^{\alpha} \lambda(x, \tau)}{\partial \tau^{\beta}} \delta u_{n}(x, \tau)(d \tau)^{\alpha}
\end{aligned}
$$

Similarly, we can get the coefficients of $\delta u_{n}$ to zero:

$$
1+\left.\lambda(x, \tau)\right|_{\tau=t}=0, \quad \frac{\partial^{\alpha} \lambda(x, \tau)}{\partial \tau^{\alpha}}=0
$$

The generalized Lagrange multiplier can be identified by the above equations,

$$
\lambda(x, t)=-1
$$

Substituting Eq. (56) into Eq. (53) produces the iteration formulation as follows

$$
\begin{aligned}
& u_{n+1}(x, t)=u_{n}(x, t)-\frac{1}{\Gamma(\alpha+1)} \\
& \int_{0}^{t}\left\{\begin{array}{l}
\frac{\partial^{\alpha} u_{n}(x, \tau)}{\partial \tau^{\alpha}}+\frac{\partial u_{n}(x, \tau)}{\partial x} \\
-\frac{\tau^{1-\alpha}}{\Gamma(2-\alpha)} \sin x-\tau \cos x
\end{array}\right\}(d \tau)^{\alpha}
\end{aligned}
$$

Taking the initial value $u_{0}(x, t)=u_{0}(x, 0)=0$, we can derive

$$
\begin{aligned}
& u_{1}(x, t)=u_{0}(x, t)-\frac{1}{\Gamma(\alpha+1)} \\
& \int_{0}^{t}\left\{\begin{array}{l}
\frac{\partial^{\alpha} u_{0}(x, \tau)}{\partial \tau^{\alpha}}+\frac{\partial u_{0}(x, \tau)}{\partial x} \\
-\frac{\tau^{1-\alpha}}{\Gamma(2-\alpha)} \sin x-\tau \cos x
\end{array}\right\}(d \tau)^{\alpha} \\
& u_{1}(x, t)=t \sin x+\frac{t^{1+\alpha}}{\Gamma(2+\alpha)} \cos x,
\end{aligned}
$$

By the similar operations, we have

$$
\begin{aligned}
& u_{2}(x, t)=t \sin x+\frac{t^{1+2 \alpha}}{\Gamma(2+2 \alpha)} \sin x, \\
& u_{3}(x, t)=t \sin x-\frac{t^{1+3 \alpha}}{\Gamma(2+3 \alpha)} \cos x, \\
& u_{4}(x, t)=t \sin x-\frac{t^{1+4 \alpha}}{\Gamma(2+4 \alpha)} \sin x, \\
& u_{5}(x, t)=t \sin x+\frac{t^{1+5 \alpha}}{\Gamma(2+5 \alpha)} \cos x,
\end{aligned}
$$

Canceling the noise terms and keeping the non-noise terms yield the exact solution of Eq. (51) given by

$$
u(x, t)=t \sin x,
$$

Which is easily confirmed. This formally proved right in [18].

\section{Example 6}

We consider the following one-dimensional linear inhomogeneous fractional Klein-Gordon equation [37] where $1<\alpha \leq 2, t>0, x \in R$

$$
D_{t}^{\alpha} u-u_{x x}+u=6 x^{3} t+\left(x^{3}-6 x\right) t^{3},
$$

With initial conditions

$$
u(x, 0)=0, u_{t}(x, 0)=0 .
$$

Construction the following functional:

$$
\begin{aligned}
& u_{n+1}(x, t)=u_{n}(x, t)+\frac{1}{\Gamma(\alpha+1)} \\
& \int_{0}^{t} \lambda(x, \tau)\left\{\begin{array}{l}
\frac{\partial^{\alpha} u_{n}(x, \tau)}{\partial \tau^{\alpha}}-\frac{\partial^{2} u_{n}(x, \tau)}{\partial x^{2}}+u_{n}(x, \tau) \\
-6 x^{3} \tau-\left(x^{3}-6 x\right) \tau^{3}
\end{array}\right\}(d \tau)^{\alpha}
\end{aligned}
$$

We have

$$
\begin{aligned}
& \delta u_{n+1}(x, t)=\delta u_{n}(x, t)+\frac{1}{\Gamma(\alpha+1)} \\
& \delta \int_{0}^{t} \lambda(x, \tau)\left\{\begin{array}{l}
\frac{\partial^{\alpha} u_{n}(x, \tau)}{\partial \tau^{\alpha}}-\frac{\partial^{2} u_{n}(x, \tau)}{\partial x^{2}} \\
+u_{n}(x, \tau)-6 x^{3} \tau-\left(x^{3}-6 x\right) \tau^{3}
\end{array}\right\}(d \tau)^{\alpha} \\
& =\delta u_{n}+\left.\lambda \delta u_{n}\right|_{\tau=t}-\frac{1}{\Gamma(\alpha+1)} \int_{0}^{t} \frac{\partial^{\alpha} \lambda(x, \tau)}{\partial \tau^{\beta}} \delta u_{n}(x, \tau)(d \tau)^{\alpha}
\end{aligned}
$$


Citation: Merdan M (2012) A Numeric-Analytic Method for Fractional Order Nonlinear PDE's With Modified Riemann-Liouville Derivative by Means of Fractional Variational Iteration Method. J Appl Computat Math 1:113. doi:10.4172/2168-9679.1000113

Page 6 of 7

$$
1+\left.\lambda(x, \tau)\right|_{\tau=t}=0, \quad \frac{\partial^{\alpha} \lambda(x, \tau)}{\partial \tau^{\alpha}}=0
$$

The generalized Lagrange multiplier can be identified by the above equations,

$$
\lambda(x, t)=-1 .
$$

substituting Eq. (66) into Eq. (63) produces the iteration formulation as follows

$$
\begin{aligned}
& u_{n+1}(x, t)=u_{n}(x, t)-\frac{1}{\Gamma(\alpha+1)} \\
& \int_{0}^{t}\left\{\begin{array}{l}
\frac{\partial^{\alpha} u_{n}(x, \tau)}{\partial \tau^{\alpha}}-\frac{\partial^{2} u_{n}(x, \tau)}{\partial x^{2}}+u_{n}(x, \tau) \\
-6 x^{3} \tau-\left(x^{3}-6 x\right) \tau^{3}
\end{array}\right\}(d \tau)^{\alpha}
\end{aligned}
$$

Taking the initial value $u_{0}(x, t)=u_{0}(x, 0)=0$, we can derive

$$
\begin{aligned}
& u_{1}(x, t)=u_{0}(x, t)-\frac{1}{\Gamma(\alpha+1)} \\
& \int_{0}^{t}\left\{\begin{array}{l}
\frac{\partial^{\alpha} u_{0}(x, \tau)}{\partial \tau^{\alpha}}-\frac{\partial^{2} u_{0}(x, \tau)}{\partial x^{2}}+u_{0}(x, \tau) \\
-6 x^{3} \tau-\left(x^{3}-6 x\right) \tau^{3}
\end{array}\right\}(d \tau)^{\alpha} \\
& =\frac{6 x^{3} t^{1+\alpha}}{\Gamma(2+\alpha)}+\frac{6\left(x^{3}-6 x\right) t^{3+\alpha}}{\Gamma(4+\alpha)},
\end{aligned}
$$

By the similar operations, we have

$$
\begin{aligned}
u_{2}(x, t)= & \frac{6 x^{3} t^{1+\alpha}}{\Gamma(2+\alpha)}+\frac{6\left(x^{3}-6 x\right) t^{3+\alpha}}{\Gamma(4+\alpha)}+\frac{36 x t^{1+2 \alpha}}{\Gamma(2+2 \alpha)}+\frac{36 x t^{3+2 \alpha}}{\Gamma(4+2 \alpha)} \\
& -\frac{6 x^{3} t^{1+2 \alpha}}{\Gamma(2+2 \alpha)}-\frac{6\left(x^{3}-6 x\right) t^{3+2 \alpha}}{\Gamma(4+2 \alpha)},
\end{aligned}
$$

From Eq. (79), variational iteration method for give the following approximations:

$$
\begin{aligned}
u(x, t)= & x^{3} t^{3}+\frac{6\left(x^{3}-6 x\right) t^{5}}{3 !}+\frac{36 x t^{5}}{5 !}-\frac{6 x^{3} t^{5}}{5 !}+\frac{36 x t^{7}}{7 !} \\
& -\frac{6\left(x^{3}-6 x\right) t^{3+2 \alpha}}{7 !}+\cdots
\end{aligned}
$$

Canceling the noise terms and keeping the non-noise terms in (70) yield the exact solution of Eq. (61), for the special case $\alpha=2$,

$$
u(x, t)=x^{3} t^{3}
$$

Which is easily verified.

\section{Conclusions}

The VIM has been successfully applied to derive explicit numerical solutions for nonlinear problems and ordinary, partial, fractional, integral equations. In this paper, we have discussed modified variational iteration method having integral w.r.t. $(d \tau)^{\alpha}$ used for the first time by Jumarie. The obtained results indicate that this method is powerful and meaningful for solving the nonlinear fractional differential equations. Six examples indicate that the results of variational iteration method having integral w.r.t. $(d \tau)^{\alpha}$ are in excellent agreement with those obtained by classical VIM, GDTM, HPM and Adomian decomposition method which is available in the literature.

\section{References}

1. Oldham KB, Spanier J (1974) The Fractional Calculus. Academic Press, New York.

2. Miller KS, Ross B (1993) An Introduction to the Fractional Calculus and Fractional Differential Equations. Wiley, New York.

3. Podlubny I (1999) Fractional Differential Equations. Academic Press, New York

4. Kilbas AA, Srivastava HM, Trujillo (2006) JJ Theory and Applications of Fractional Differential Equations. Elsevier, Amsterdam.

5. Samko SG, Kilbas AA, Marichev OI (1993) Fractional Integrals and Derivatives: Theory and Applications. Gordon and Breach, Yverdon.

6. Caputo $M(1967)$ Linear models of dissipation whose $Q$ is almost frequency independent. Geophys J Roy Astr Soc 13: 529.

7. Merdan M, Gökdoğan A, Yıldırım A, Tauseef Syed, Mohyud-Din (2012) ST Numerical simulation of fractional Fornberg-Whitham equation by differential transformation method. Abstract and Applied Analysis.

8. Merdan M (2012) On the Solutions Fractional Riccati Differential Equation with Modified Riemann-Liouville Derivative. International Journal of Differential Equations.

9. He JH (1999) Variational iteration method- a kind of non-linear analytica technique: Some examples. Int J Nonlinear Mech 34: 699-708.

10. He JH (1999) Some applications of nonlinear fractional differential equations and their approximations. Bull Sci Technol 15: 86-90.

11. Momani S, Yildirim A (2010) Analytical approximate solutions of the fractional convection-diffusion equation with nonlinear source term by He's homotopy perturbation method. Int J Comput Math 87: 1057-1065.

12. Yildirim A, Kocak H (2009) Homotopy perturbation method for solving the space-time fractional advection-dispersion equation. Adv Water Resour 32 1711-1716.

13. Yildirim A, Gulkanat $Y$ (2010) Analytical approach to fractional ZakharovKuznetsov equations by He's homotopy perturbation method. Commun Theor Phys 53: 1005.

14. El-Shahed M (2005) Application of He's homotopy perturbation method to Volterra's integro-differential equation. Int J Nonlinear Sci Numer Simul 6: 163 168

5. Siddiqui AM, Mahmood R, Ghori QK (2006) Thin film flow of a third grade fluid on a moving belt by He's homotopy perturbation method. Int J Nonlinear Sci Numer Simul 7: 7-14

16. Yildirim A (2010) Analytical approach to fractional partial differential equations in fluid mechanics by means of the homotopy perturbation method. Internat $J$ Numer Methods Heat Fluid Flow 20: 186-200.

17. Das S, Gupta PK, Barat S (2010) A note on fractional Schrödinger equation Nonlinear Sci Lett A 1: 91-94.

18. Yildirim A (2009) An algorithm for solving the fractional nonlinear Schrödinge equation by means of the homotopy perturbation method. Int J Nonlinear Sci Numer Simul 10: 445-451.

19. Al-Khaled K, Momani S (2005) An approximate solution for a fractiona diffusion-wave equation using the decomposition method. Appl Math Comput 165: 473-483.

20. Mainardi F, Luchko Y, Pagnini G (2001) The fundamental solution of the space time fractional diffusion equation. Frac Calc Appl Anal 4: 153-192.

21. Hanyga A (2002) Multidimensional solutions of time-fractional diffusion-wave equations. Proc R Soc Lond A 458: 933-957.

22. Huang F, Liu $F(2005)$ The time fractional diffusion and fractional advectiondispersion equation. ANZIAM 46: 1-14.

23. Huang F, Liu $F(2005)$ The fundamental solution of the space-time fractiona advection-dispersion equation. J Appl Math Comput 18: 339-350. 
Citation: Merdan M (2012) A Numeric-Analytic Method for Fractional Order Nonlinear PDE's With Modified Riemann-Liouville Derivative by Means of Fractional Variational Iteration Method. J Appl Computat Math 1:113. doi:10.4172/2168-9679.1000113

24. Momani S (2005) Analytic and approximate solutions of the space- and timefractional telegraph equations. Appl Math Comput 170: 1126-1134.

25. Momani S (2005) An explicit and numerical solutions of the fractional KdV equation. Math Comput Simul 70: 110-118.

26. Debnath L, Bhatta D (2004) Solutions to few linear fractional inhomogeneous partial differential equations in fluid mechanics. Frac Calc Appl Anal 7: 21-36.

27. Jumarie G (1993) Stochastic differential equations with fractional Brownian motion input. Int J Syst Sci 6: 1113-1132.

28. Jumarie G (2006) New stochastic fractional models for Malthusian growth the Poissonian birth process and optimal management of populations. Math Comput Model 44: 231-254.

29. Jumarie G (2009) Laplace's transform of fractional order via the Mittag-Leffler function and modified Riemann-Liouville derivative. Appl Math Lett 22: 16591664.

30. Jumarie G (2009) Table of some basic fractional calculus formulae derived from a modified Riemann-Liouvillie derivative for nondifferentiable functions. App Math Lett 22: 378-385.

31. Jumarie G (2005) On the solution of the stochastic differential equation of exponential growth driven by fractional Brownian motion. Appl Math Lett 18: 817-826.
32. Merdan M, Mohyud-Din ST (2011) A New Method for Time-fractional CoupledKDV Equations with Modified Riemann-Liouville Derivative. Studies in Nonlinear Sciences 2: 77-86.

33. Roul P (2011) Numerical solutions of time fractional degenerate parabolic equations by variational iteration method with Jumarie-modified RiemannLiouville derivative. Math Method Appl Sci 34: 1025-1035.

34. Luchko AY, Groneflo R (1998) The initial value problem for some fractional differential equations with the Caputo derivative. Preprint series A08_98, Fachbreich Mathematik und Informatik, Freic Universitat Berlin.

35. Naeem Faraz, Yasir Khan, Hossein Jafari, Ahmet Yildirim , Madani M (2011) Fractional variational iteration method via modified Riemann-Liouville derivative. Journal of King Saud University (Science) 23: 413-417.

36. Dehghan M, Manafian J, Saadatmandi (2010) A Solving nonlinear fractiona partial differential equations using the homotopy analysis method. Numer Meth Partial Diff Equ 26: 448-479.

37. Odibat Z , Momani S (2009) The variational iteration method: An efficien scheme for handling fractional partial differential equations in fluid mechanics. Comput Math Appl 58: 2199-2208. 